“ (C) 2017 IEEE. Personal use of this material is permitted. Permission from IEEE must be obtained for all other uses, in any current or future media, including

reprinting/republishing this material for advertising or promotional purposes, creating new collective works, for resale or redistribution to servers or lists, or reuse of any copyrighted component of this work in other works." 


\title{
Wideband Matching of Full-Wavelength Dipole with Reflector for Base Station
}

\author{
Can Ding, Bevan Jones, Y. Jay Guo, and Pei-Yuan Qin
}

\begin{abstract}
This paper introduces a wide-band hybrid feeding method for full-wavelength dipole antennas with a reflector. A full-wavelength dipole is designed to cover the band from $698 \mathrm{MHz}$ to $960 \mathrm{MHz}$ for cellular base station applications. Its matching circuit consists of a tripletuned circuit and a quasi-quarter-wavelength impedance transformer. The proposed matching circuit can provide balanced feeding as a balun and has a compact size. The working mechanism and a complete design scheme of the proposed matching circuit are elaborated. The matching circuit is designed and optimized using a circuit theory model and then physically realized using microstrip lines based on full-wave simulation. The measured reflection coefficient $\left|S_{11}\right|$ is $<-14 \mathrm{~dB}$ across the entire band from $698 \mathrm{MHz}$ to $960 \mathrm{MHz}$, exhibiting a bandwidth of $32 \%$. This is the first time that a wideband centre-fed full-wavelength dipole is proposed.
\end{abstract}

Index Terms-Full-wavelength Dipole, Wideband, Hybrid Matching Circuit, Base station.

\section{INTRODUCTION}

$\mathbf{T}$ HE dipole antenna is one of the simplest and most widely used antennas in wireless communication and sensing. Dipoles have two arms with standing waves of current flowing back and forth between their ends. For very short dipole antennas (with length < $\lambda / 10$, where $\lambda$ is the wavelength at the operating frequency), the center-fed input impedance is capacitive with a small real resistance. As the length of the dipole increases, the reactance reduces and the resistance increases. When the length of the dipole reaches approximately $\lambda / 2$, the dipole has a zero reactance and is said to be resonant. Around this resonant frequency, the dipole has a quarterperiod standing wave on each of its arms as shown in Fig. 1(a). Anti-resonance occurs when the dipole's length becomes close to $\lambda$, and thus the dipole is called a full-wavelength dipole. As shown in Fig. 1(b), in this case, there are two half-period standing waves on the dipole. This makes a full-wavelength dipole more directive than a half-wavelength dipole. While half-wavelength dipoles are popular in a variety of applications, full-wavelength dipoles are seldom used mainly due to the difficulties in matching.

Based on the simple standing wave model and neglecting the radiation, the impedance of a full-wavelength dipole at the center would be infinite. In reality, it is not infinite but can still be several hundreds or thousands of $\Omega$ s. The high resistance makes the fullwavelength dipole difficult to feed. If this issue can be solved, fullwavelength dipoles can be used in many potential applications to make use of the narrower beamwidth. In addition to the higher gain and narrower beamwidth, another significant advantage of fullwavelength dipoles is that they have a much broader bandwidth than half-wavelength dipoles of the same diameter in a similar configuration. For example, the $Q$ of the dipole considered in this work as shown in Fig. 2 is 2.8, whereas that of a half-wavelength dipole of the same diameter and height above the ground plane is 5.4. As discussed in [1], the $Q$ of an antenna is approximately inversely proportional to the bandwidth of a simply tuned antenna at

All the authors were with the Global Big Data Technologies Centre (GBDTC), University of Technology Sydney (UTS), Sydney, NSW, 2007, Australia. e-mail:(can.ding@uts.edu.au)

Manuscript received October 19, 2016; revised February 02, 2017; and June 06, 2017; accepted June 30, 2017.

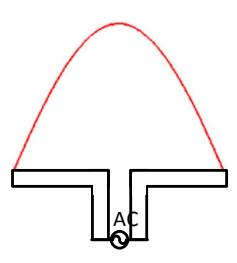

(a)

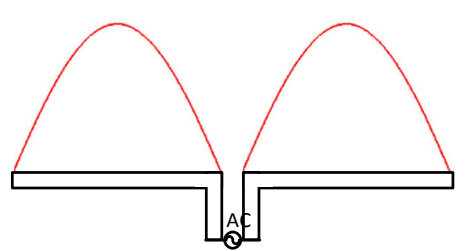

(b)
Fig. 1. Current distribution on (a) half- and (b) full-wavelength dipoles.

all frequencies. This indicates that, by simply increasing the dipole length from nearly half wavelength to nearly a wavelength, the dipole not only gets a narrower beamwidth but also a significantly widened bandwidth, on the premise that the full-wavelength dipole is well matched.

However, to the best of our knowledge, there are few published studies on full-wavelength dipoles. Offset-feeding [2-4] and dual/triple-feeding [5-8] are the only available methods published in the literature. Offset-feeding is realized by moving the feed point away from the center of the dipole. Usually, the new feed point lies on one of the arms where the current reaches the peak. However, according to $[3,4]$, the offset-feeding can deteriorate the radiation performance by introducing split lobes. Investigations in [5-8] show that a full-wavelength dipole can be better fed by employing additional feed points. But both of these methods feature high fabrication complexity. Besides, none of these work is focused on the impedance bandwidth. It is important to develop a centrefed wideband impedance matching method to realize the wideband characteristic of full-wavelength dipoles.

There are extensive studies on broadband impedance matching methods since the 1940s. Among the available matching methods, the Fano [9] and Wheeler's [10-12] single/double-tuned matching are universally useful for matching small antennas and not-sosmall antennas. In 1950, Fano [9] published his famous impedancematching equations, which related the Q-bandwidth product of a load to the maximum reflection magnitude for all levels of multiple tuning circuits. Nearly at the same time, Wheeler used a different approach to derive his single/double-tuned matching networks [10, 11]. Later Lopez's studies $[13,14]$ showed that the Fano and Wheeler equations were in exact agreement for single and double-tuned matching (Fano $\mathrm{n}=1$ and 2 cases), and illustrated the key features of the optimum single/double-tuned matching method. Then triple-tuned matching was developed by Simpson [15] based on Wheeler's doubletuned matching network. But the practical significance of triple-tune matching is marginal since it increases the design complexity and size and it does not provide much benefit over double-tune matching $[16,17]$. The Wheeler and Fano matching methods were initially targeted at small antennas that can be seen as a small resistance in series with a small capacitance or inductance. But the concept of Wheeler's double-tuned matching is also widely used for halfwavelength dipoles and monopoles, where the dipole themselves are series resonators. 


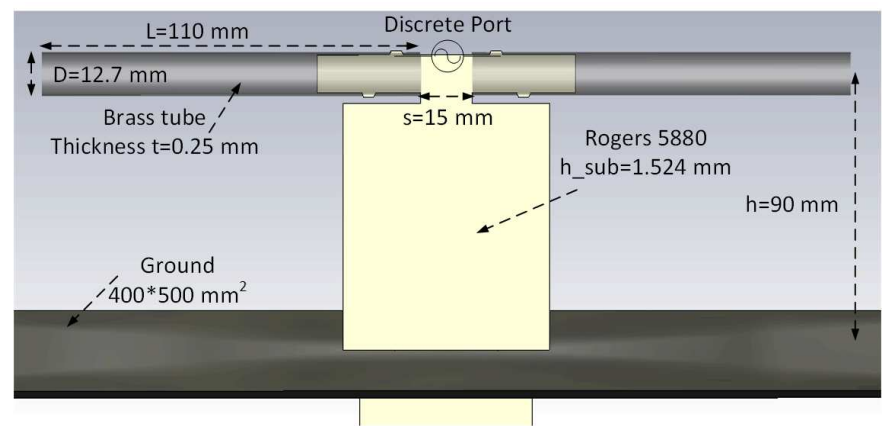

Fig. 2. Dipole configuration.

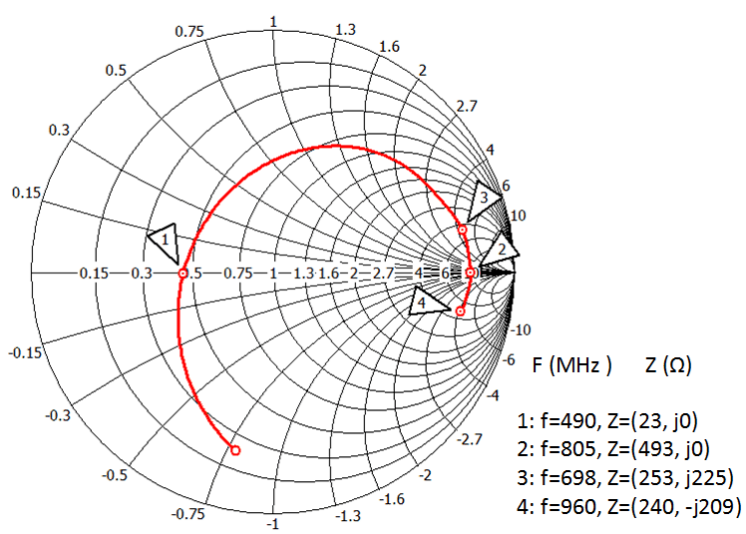

Fig. 3. Calculated input impedance of the dipole without matching network.

In this paper, we propose a hybrid triple-tuned matching circuit with an integrated balun for anti-resonant full-wavelength dipoles. A straight-wire full-wavelength dipole is designed to demonstrate the proposed matching method. The measurement result shows that a wideband matching of $32 \%$ from $698 \mathrm{MHz}$ to $960 \mathrm{MHz}$ is achieved with $\left|S_{11}\right|<-14 \mathrm{~dB}$.

\section{DIPOLE AND MATCHING CIRCUIT DESIGN}

\section{A. Full-wavelength dipole}

Fig. 2 shows the full-wavelength dipole which is to be matched. The target band is from $698 \mathrm{MHz}$ to $960 \mathrm{MHz}$, which is of interest for cellular base station applications. Brass tubes are employed as the dipole arms as they are convenient for experimental implementation. The diameter and thickness of the brass tubes are $D=12.7 \mathrm{~mm}$ and $t=0.25 \mathrm{~mm}$, respectively. The two arms have the same length of $L=110 \mathrm{~mm}$ and are separated by a distance of $s=15 \mathrm{~mm}$. The dipole is placed $90 \mathrm{~mm}$ above a conductive ground, which is approximately $\lambda_{0} / 4$, where $\lambda_{0}$ is the wavelength at the center frequency $(818 \mathrm{MHz})$ of the target band in the open air. These dimensions are chosen so that the dipole has the first anti-resonance near $818 \mathrm{MHz}$. Rogers 5880 substrate (dielectric constant $\epsilon=2.2$, loss tangent $\tan \delta=0.0009$, and thickness $h \_s u b=1.524 \mathrm{~mm}$ ) is used to support the brass tubes and later, the matching circuit. Fig. 3 shows the calculated input impedance of the dipole excited by a lumped port between the tubes. According to the calculated result, the proposed dipole has the first resonance and anti-resonance at $490 \mathrm{MHz}$ and $805 \mathrm{MHz}$, where the dipole operates as a half- and full-wavelength dipole, respectively.

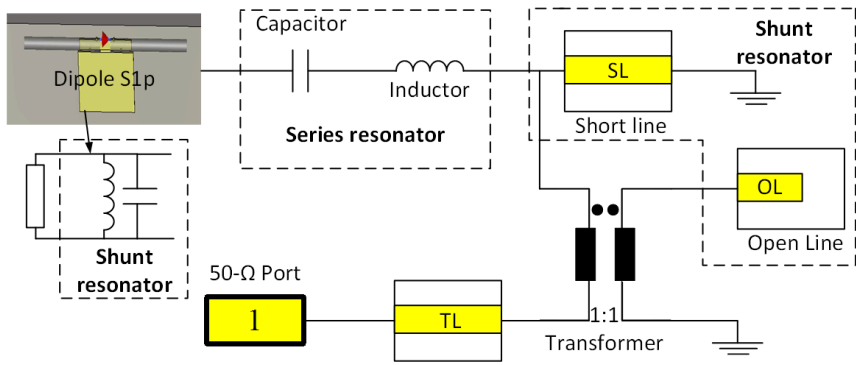

(a)

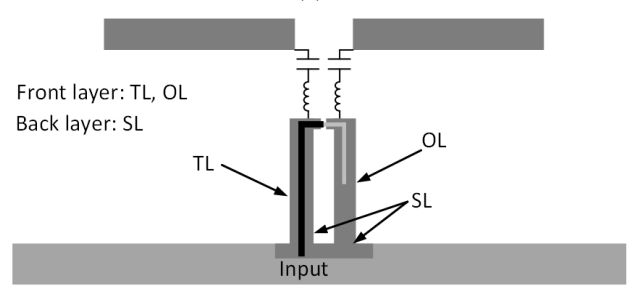

(b)

Fig. 4. Configuration of the matching circuit for the full-wavelength dipole: (a) non-differential ADS version and (b) microstrip version.

\section{B. Matching network design and optimization}

The impedance of the dipole near its anti-resonant frequency can be approximated well by a parallel resonant R-L-C circuit where $\mathrm{R}$ represents the radiation resistance. The idea of impedance matching described in this work is to design a ladder-type band-pass filter using serial and shunt resonant L-C circuits as shown in Fig. 4(a). The first resonator in this arrangement is the shunt $\mathrm{L}-\mathrm{C}$ of the equivalent circuit of the dipole. Following this shunt resonator, we require a series resonator. This is implemented as a pair of series L-C resonant circuits shown in Fig. 4(b). They are split as shown to maintain symmetry and preserve balance. Note that the values of the inductances in the balanced configurations shown in Fig. 4(b) are half of those in unbalanced representation shown in Fig. 4(a) and the capacitances are double. The next resonator of the ladder filter is provided by the balanced tracks in microstrip implementation of the balun. This is a shorted quarter-wavelength line which provides a balanced shunt resonator. This basically concludes the implementation of the filter. Finally, a segment of transmission line (TL) nearly quarterwavelength long is used to transform the high impedance presented at the balun gap to $50 \Omega$. The quasi-quarter-wavelength TL transformer is not sufficiently wideband for this application but the series open circuit line impedance OL helps to compensate the quarter-wavelength line transformer. The subsequent optimization allows compensating elements to improve the transformer's performance. Note that in microstrip implementation as shown in Fig. 4(b), the microstrip line OL and TL are printed on the front layer, while the SL, inductors, and capacitors are placed on the back layer of the substrate. This way, the SL can be used as the ground for the OL and TL, resulting in a modified Marchand balun [20-22].

The optimization was conducted in ADS using the configuration shown in Fig. 4(a). The series connection of the balun and OL is achieved with the artifice of an ideal 1:1 transformer. After optimizing all the components used in the matching circuit, the full-wavelength dipole is successfully matched across the target band with $\left|S_{11}\right|<$ $-15 \mathrm{~dB}$. The input impedance in the Z-smith chart after matching is shown in Fig. 5. Fig. 5 also gives the optimized values of the lumped components and the characteristic impedance $(\mathrm{Z})$ and the length $(\mathrm{L})$ of the transmission lines.

The real and imaginary parts of the input impedances at different 


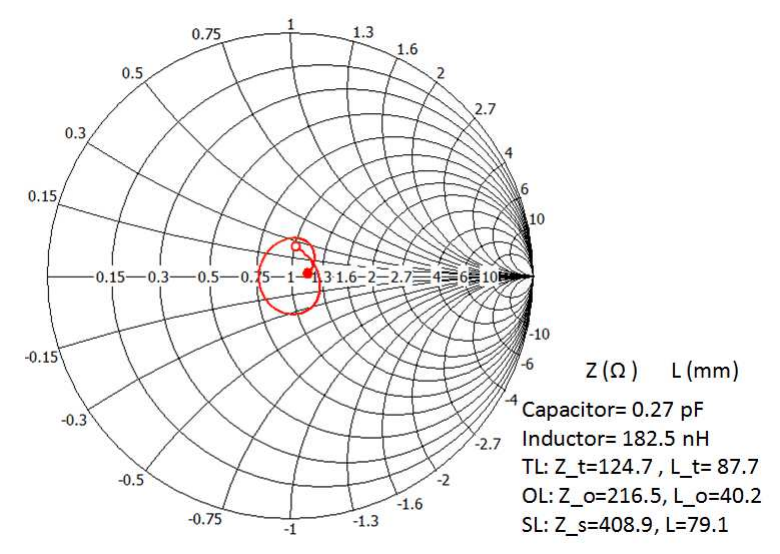

Fig. 5. Calculated input impedance after matching.

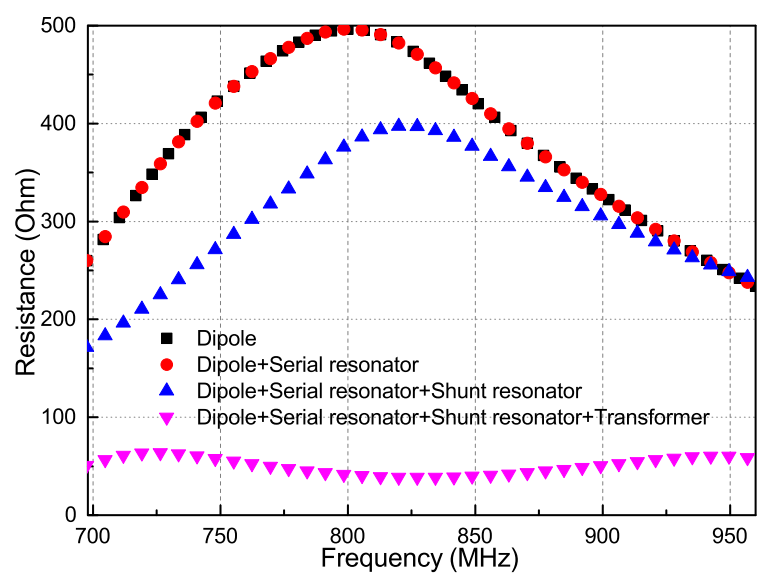

(a)

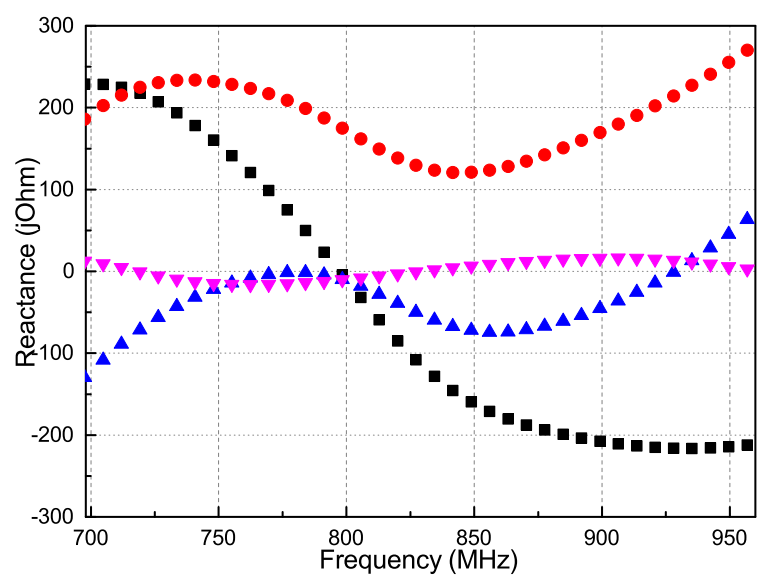

(b)

Fig. 6. (a) Real and (b) imaginary parts of the input impedances when the antenna is matched with different stages of tuning components.

nodes in the matching circuit are plotted in Fig. 6. As shown in Fig. 6(a), the dipole without a matching network has a quite high resistance within the designed band. The series resonator do not affect the resistance at resonance. The shunt resonator slightly reduces the high resistance of the dipole in the required band. The quasi-quarterwavelength transformer is the key component that transforms the resistance to $50-\Omega$. According to Fig. 6 , the variation of reactance of the dipole is mainly mitigated by the two resonators. The first serial-resonator significantly alleviates the fluctuation of the reactance

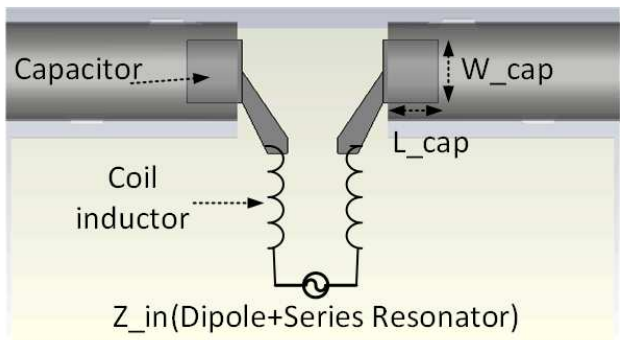

Fig. 7. Realization of the series resonator.

across the operation band. Then the second shunt resonator translates the reactance from inductive to nearly zero. Note that there are other combinations of the $\mathrm{L}-\mathrm{C}$ tuning circuits that are able to cancel the reactance within the target band. The line transformer TL also contributes a little in cancelling the reactance. After optimization, the TL is not exactly a quarter-wavelength long. The OL in series with the TL compensates the narrow band performance of the line transformer TL.

\section{MATCHING CIRCUIT PHYSICAL IMPLEMENTATION}

To validate the proposed matching circuit in the previous section, a 3D model should be built and the result should be checked in a full-wave simulation environment. In doing so we observed some discrepancies between the electrical characteristics of the ideal components in circuit theory and those of the actual realized components in full-wave simulation. Although the discrepancies are small, they can add up and lead to degraded matching performance. Therefore, proper implementations and adjustments of all the components are essential. In this work, the employed matching components were implemented one by one. After each realization of the components, the input impedance of the dipole was re-calculated. Based on the obtained results, the rest of the matching circuit was then re-optimized in circuit theory model to compensate the discrepancies. Detailed implementation methods for all the components are described as follows.

\section{A. Series resonator realization}

The series resonator is realized using lumped capacitors and inductors. The capacitors are realized by inserting a small rectangle copper strip into each of the brass tubes as shown in Fig. 7. The capacitance provided by the pads can be adjusted by changing their width ( $W_{-}$cap) and length ( $\left.L \_c a p\right)$. An easy way to get the value of the capacitance introduced by the pads is to run a full-wave simulation considering a pad together with the brass tube as a coaxial line. From HFSS or CST, we can easily get the characteristic impedance $\left(Z=\sqrt{L_{0} / C_{0}}\right)$ and the propagation constant $\left(\beta=\omega \sqrt{L_{0} C_{0}}\right)$ of the coaxial line, where $L_{0}$ and $C_{0}$ are the inductance and capacitance per unit length. Then the capacitance provided by the strip can be calculated as $C_{\text {strip }}=C_{0} \times L_{-}$cap. Note that the strip should have a smaller $L \_$cap and larger $W \_$cap to minimize the resultant inductance.

The inductance is provided by coil wires as shown in Fig. 7. Two strip lines printed on the substrate are used to connect the coils with capacitors. In full-wave simulation, the inductors are still assumed to be ideal. In experiment, the inductors were made by twining coils on a dielectric screw. The initial dimensions of the coil inductors were obtained from the coil-based inductance calculator online. Then we measured the inductance in VNA (Vector Network Analyser) and adjusted the dimensions to obtain the right value. 


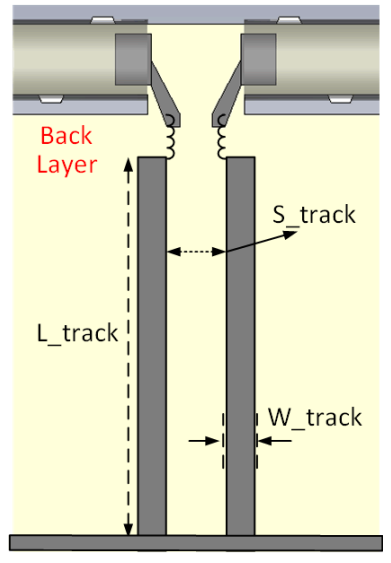

Fig. 8. Realization of the Balun.

Due to the fact that in circuit theory the capacitors and inductors are assumed to be directly connected to the dipole, while in reality the capacitors are coupled to the tubes in a more complicate way through the distributed electro-magnetic fields, there is a discrepancy between the ideal and actual input impedance $Z_{i n}$ (Dipole + Series Resonator). But the dipole can still be matched by optimizing the remaining elements in circuit model based on the actual input impedance $Z_{\text {in }}$ (Dipole + Capacitors). Note that in the revised matching circuit, the OL used as a part of the shunt resonator in Fig. 4(a) is replaced by a second short line (SL2). This is due to the fact that the required length when using SL is shorter than than when using OL to get the right impedance.

\section{B. Shunt resonator and balun realization}

The SL shown in Fig. 4(a) also functions as the balun. Fig. 8 shows the layout of the balun realized by a pair of metallic tracks shorted to the ground. Meanwhile, they also function as the ground planes for the TL and SL2 printed on the opposite side of the substrate. The two parallel tracks can be considered as a pair of coupled lines on substrate without a ground plane (with their ground plane infinitely far away). The inductance- and capacitance-matrix of the coupled lines can be calculated using the method described in [18]. They are then used to derive the odd-mode characteristic impedance and phase velocity of the coupled lines. In this work, $W_{-}$track and $S_{-}$track are selected to be 5 and $8 \mathrm{~mm}$, respectively, to achieve the odd-mode characteristic impedance of $250 \Omega$ as required.

The remaining components in the matching circuit including TL and SL2 are realized by conventional microstrip lines. They are printed on the front layer of the substrate, using the metallic tracks (SL) as their ground plane. Their line width and length can be calculated from the thickness and dielectric constant of the used substrate and the target characteristic impedances.

The layout of the entire matching circuit is shown in Fig. 9. Fig. 9 (a) is a perspective view of the dipole illustrating both the two layers with the substrate is set transparent. The TL and SL2 are printed on the front layer of the substrate while the capacitors, inductors, and the balun (SL) are placed on the back layer of the substrate, as shown in Fig. 9(b). Note that the SL, SL2, and TL are bent towards the plane of symmetry at the top ends, and a gap with the width of only $1 \mathrm{~mm}$ is left between the two metallic tracks as shown in Fig. 9. Optimal cuts are applied to all the bends to minimize reflection. Overall fine tuning of the dimensions of the microstrip lines and metallic tracks are then conducted in full-wave simulations to obtain an optimal performance. The optimized dimension values of the matching circuit are listed in

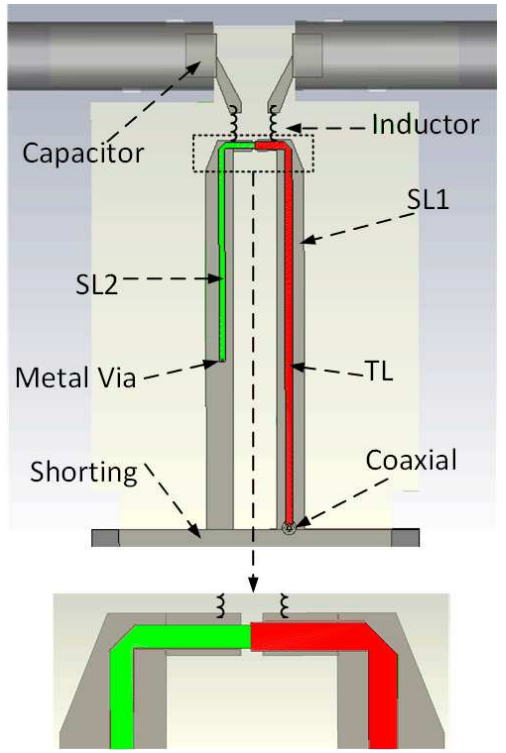

(a)

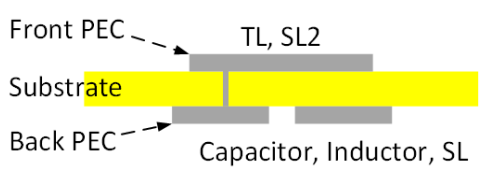

(b)

Fig. 9. (a) Perspective view and (b) cross-section view of the realized matching circuit.

TABLE I

Optimized Dimension VAlues of The Matching Circuit

\begin{tabular}{|l|l|l|}
\hline Parameter & Value & Description \\
\hline$L \_c a p$ & $5.5 \mathrm{~mm}$ & Length of the inserted capacitors \\
\hline W_cap & $8 \mathrm{~mm}$ & Width of the inserted capacitors \\
\hline$I$ & $14.75 \mathrm{nH}$ & Inductance of the lumped inductor \\
\hline L_track & $73 \mathrm{~mm}$ & Length of the metallic tracks \\
\hline W_track & $5 \mathrm{~mm}$ & Width of the metallic tracks \\
\hline$L \_T L$ & $74.48 \mathrm{~mm}$ & Length of the TL \\
\hline W_TL & $1.35 \mathrm{~mm}$ & Width of the TL \\
\hline$L \_S L$ & $44.52 \mathrm{~mm}$ & Length of the SL2 \\
\hline W_SL & $1.08 \mathrm{~mm}$ & Width of the SL2 \\
\hline
\end{tabular}

Table I. Note that the lengths of the TL, SL1, and SL2 in the table are the total lengths of the $\Gamma$-shaped microstrip lines.

\section{Results}

The optimized model shown in Fig. 9 was fabricated and tested. The prototype is pictured in Fig. 10. The dipole is placed on a ground plane with the size of $400 \times 500 \mathrm{~mm}^{2}\left(1.09 \lambda_{0} \times 1.36 \lambda_{0}\right)$. The dipole is fed by a coaxial cable located beneath the ground. The simulated and measured reflection coefficients $\left|S_{11}\right|$ of the matched dipole are plotted in Fig. 11. It is observed that they agree well with each other. According to the measured results, the full-wavelength dipole has an excellent impedance match $\left(\left|S_{11}\right|<-14 d B\right)$ across the target band from $698 \mathrm{MHz}$ to $960 \mathrm{MHz}$, exhibiting a wide bandwidth of $32 \%$. Figs. 12(a) and 12(b) show the simulated radiation patterns in the E- and H-planes, respectively, at 698, 785, 873, and $960 \mathrm{MHz}$. The radiation patterns were not measured since they are not the major concern in this work. From the figure we notice that the patterns show some asymmetry in the E-plane at higher frequencies. This is 


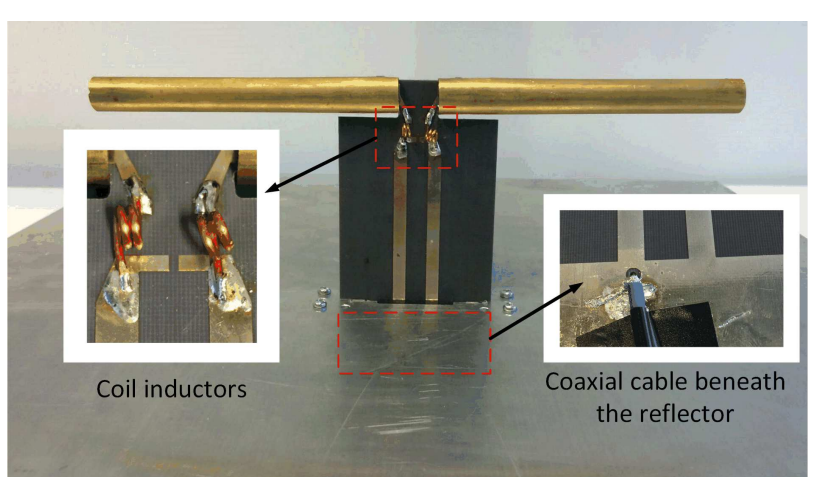

Fig. 10. Fabricated prototype of the full-wavelength dipole.

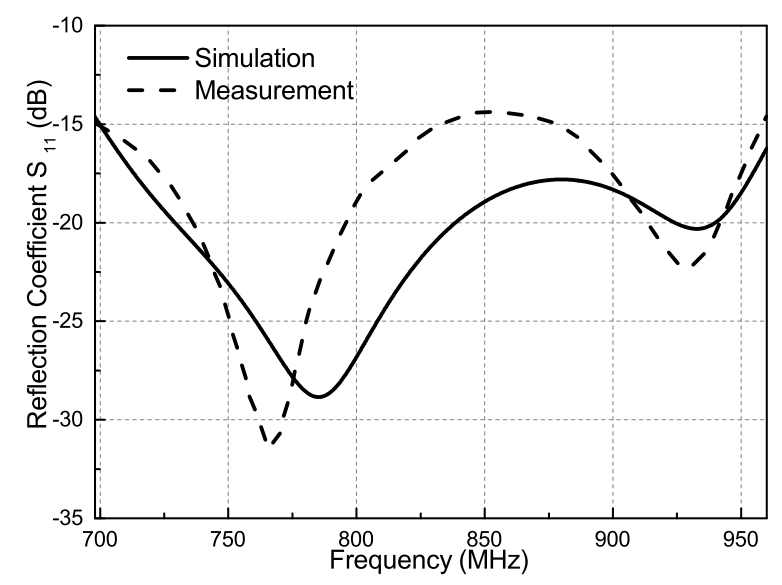

Fig. 11. Simulated and measured $\left|S_{11}\right|$ of the full-wavelength dipole with a matching circuit.

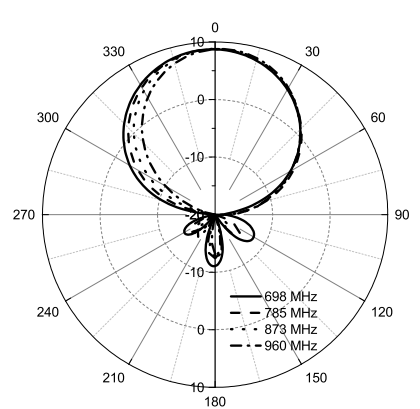

(a)

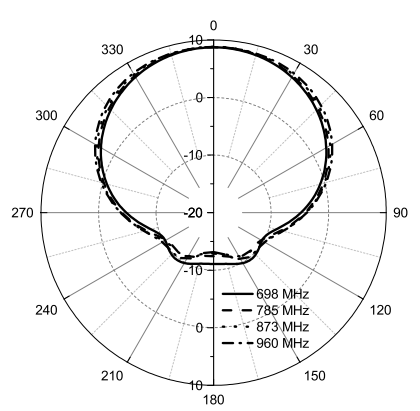

(b)
Fig. 12. Simulated radiation patterns in the (a) E- and (b) H-plane at 698, 785, 873, and $960 \mathrm{MHz}$.

attributed to the asymmetry of the microstrip balun leading to slightly unbalanced currents on the arms of the dipole. Despite this problem, the radiation patterns are quite stable in both the E- and H-planes within the operation band. According to the simulation results, the realized gain of the dipole varies from 8.5 to $8.7 \mathrm{dBi}$, and the crosspolarization level are $<-25 \mathrm{~dB}$ across the entire band.

Moreover, a comparison between the performance of the fullwavelength dipole proposed in this work and a half-wavelength dipole in a similar configuration is presented in Table II. The halfwavelength dipole uses the same brass tubes (shown in Fig. 2) with a shorter length $(\mathrm{L}=55 \mathrm{~mm}, \mathrm{~s}=15 \mathrm{~mm})$ as its dipole arms. The length of the brass tubes is optimized so that the half-wavelength dipole resonates at the same frequency as that of the full-wavelength dipole. Both the dipoles are placed above an infinite ground plane
TABLE II

COMPARISON BETWEEN THE FULL- AND HALF-WAVELENGTH DIPOLE

\begin{tabular}{|l|l|l|}
\hline Parameter & Full-wave dipole & Half-wave dipole \\
\hline Dipole length & $235 \mathrm{~mm}$ & $125 \mathrm{~mm}$ \\
\hline Directivity & $8.1-8.5 \mathrm{dBi}$ & $7.3-8.0 \mathrm{dBi}$ \\
\hline E-plane 3-dB Beamwidth & $60^{\circ}-63^{\circ}$ & $71^{\circ}-78^{\circ}$ \\
\hline H-plane 3-dB Beamwidth & $101^{\circ}-124^{\circ}$ & $103^{\circ}-122^{\circ}$ \\
\hline Antenna Q & 2.8 & 5.4 \\
\hline
\end{tabular}

by $90 \mathrm{~mm}$. As observed in Table II, the full-wavelength dipole has a higher directivity of about $0.5 \mathrm{~dB}$ than the half-wavelength dipole on average. By constructing half-wavelength dipole arrays, the directivity can be higher and the E-plane beamwidth can be narrower. However, the size is also increased. Choosing to use the half-wavelength dipole, full-wavelength dipole, or half-wavelength dipole array is a trade-off between size, directivity, and beamwidth. The real superiorities of the full-wavelength dipole are its smaller antenna Q (wider bandwidth) compared to the half-wavelength dipole, and a simpler feed network compared to the half-wavelength dipole arrays. Note that the $\mathrm{Q}$ factors were calculated according to the method described in [19].

\section{CONCLUSION}

In this work, a wideband matching method for full-wavelength dipoles is described. The matching circuit for a conventional fullwavelength dipole is developed to demonstrate this method. Design procedures and considerations are given to provide clear guidance in the design. According to the measured results, the full-wavelength dipole has an excellent matching performance with $\left|S_{11}\right|<-14 \mathrm{~dB}$ across the entire designed band from $698 \mathrm{MHz}$ to $960 \mathrm{MHz}$ for base station applications. The antenna also features stable radiation characteristics and broadband performance compared to conventional halfwavelength dipoles. It is demonstrated that full-wavelength dipoles, which are generally considered to be difficult to match, can be well matched using a compact matching circuit. With this matching issue solved, full-wavelength dipoles can be better candidates in some applications due to their narrower beamwidth and wider bandwidth compared to half-wavelength dipoles.

\section{REFERENCES}

[1] A. D. Yaghjian and S. R. Best, "Impedance, bandwidth, and Q of antennas," IEEE Transactions on Antennas and Propagation, vol. 53, No. 4, pp. 1298-1324, April 2005.

[2] S. H. Idris and C. M. Hadzer, "Analysis of the radiation resistance and gain of a full-wave dipole," IEEE Antennas and Propagation Magazine, vol. 36, No. 5, pp. 45-47, Oct. 1994.

[3] H. Hosono, K. Shibata, K. Saegusa and T. Takano, "The radiation characteristics and current distribution of a full wavelength dipole," 2014 IEEE Antennas and Propagation Society International Symposium (APSURSI), Memphis, TN, pp. 75-76, 2014.

[4] S. P. Kosta, M. D. Singh and R. P. Agarwal, "Theoretical investigations on the centre-fed full-wave dipole antenna with feed-points displaced along the axis of the dipole," India, IEE-IERE Proceedings, vol. 8, No. 2, pp. 56-59, Apr. 1970

[5] K. A. Hmood, S. I. S. Hassan and M. F. Ain, "Design of dual feeding fullwave dipole antenna for VHF," 2006 International RF and Microwave Conference, Putra Jaya, 2006, pp. 135-139.

[6] Y. S. H. Khraisat, K. A. Hmood and A. Anwar, "Analysis of the parameters of asymmetrical dual feeding full wave dipole antenna," Microwaves, Radar and Remote Sensing Symposium, 2008, Kiev, 2008, pp. 215-217.

[7] Y. Khraisat, K. Hmood and A. Anwar, "Analysis of the radiation resistance and gain of full-wave dipole antenna for different feeding design," Journal of Electromagnetic Analysis and Applications, Vol. 4 No. 6, 2012, pp. 235-242.

[8] Y. Khraisat, K. Hmood and A. Anwar, "The current distribution of symmetrical dual and triple feeding full-wave dipole antenna," Modern Applied Science, Vol. 5, No. 6, 2011, pp. 126-132. 
[9] R. M. Fano, "Theoretical limitations on the broadband matching of arbitrary impedances," Journal of the Franklin Institute, Vol. 249, No. 2, pp. 139-154, Feb. 1950.

[10] H. A. Wheeler, "Wave-signal antenna (Hazeltine double-tuned lifesaver antenna)," US Patent 2,425,585, filed: December 13, 1943; issued August 12, 1947; Vol. 249, No. 2, pp. 139-154, Feb. 1950.

[11] H. A. Wheeler, "Wideband Impedance Matching," Wheeler Laboratories Report 418, May 1950.

[12] H. A. Wheeler, "The wide-band matching area for a small antenna," IEEE Transactions on Antennas and Propagation, Vol. 31, No. 2, pp. 364-367, Mar. 1983.

[13] A. R. Lopez, "Double-tuned impedance matching," IEEE Antennas and Propagation Magazine, Vol. 54, No. 2, pp. 109-116, Apr. 2012.

[14] A. R. Lopez, "Wheeler and Fano Impedance Matching [Antenna designer's notebook]," IEEE Antennas and Propagation Magazine, vol. 49, no. 4, pp. 116-119, Aug. 2007.

[15] T. Simpson, "Triple-Tuning Small Antennas for Increased Bandwidth [Antenna Designer's Notebook]," IEEE Antennas and Propagation Magazine, vol. 54, no. 4, pp. 178-186, Aug. 2012.

[16] A. R. Lopez, "Review of narrowband impedance-matching limitations," IEEE Antennas and Propagation Magazine, vol. 46, no. 4, pp. 88-90, Aug. 2004.

[17] A. R. Lopez, "More on narrowband impedance-matching limitations," IEEE Antennas and Propagation Magazine, vol. 46, no. 6, pp. 102-102, Dec. 2004.

[18] Cao Wei, R. F. Harrington, J. R. Mautz and T. K. Sarkar, "Multiconductor Transmission Lines In Multilayered Dielectric Media," IEEE Transactions on Microwave Theory and Techniques, vol. 32, no. 4, pp. 439-450, Apr. 1984.

[19] J. Audet, "Q Calculations of L-C Circuits and Transmission Lines: A Unified Approach," QEX Magazine, pp. 43-51, Sep. 2006.

[20] N. Marchand, "Transmission Lines Conversation Transformers," Electronics, Vol. 17, no. 12, pp. 142-145, Dec. 1944.

[21] T. G. Ma, C. C. Wang and C. H. Lai, "Miniaturized Distributed Marchand Balun Using Coupled Synthesized CPWs," IEEE Microwave and Wireless Components Letters, vol. 21, no. 4, pp. 188-190, April 2011.

[22] C. H. Lin, C. H. Wu, G. T. Zhou and T. G. Ma, "General Compensation Method for a Marchand Balun With an Arbitrary Connecting Segment Between the Balance Ports," IEEE Transactions on Microwave Theory and Techniques, vol. 61, no. 8, pp. 2821-2830, Aug. 2013. 